\title{
Protection against Clostridium difficile infection in a hamster model by oral vaccination using flagellin FliC-loaded pectin beads.
}

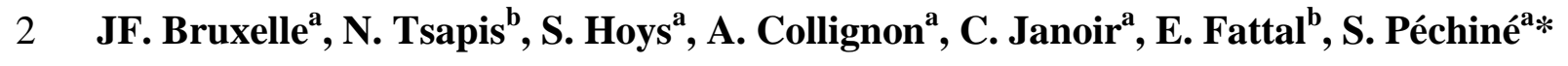

3 a EA4043 Unités Bactéries Pathogènes et Santé (UBaPS), Univ. Paris-Sud, Université Paris-Saclay,

4 Châtenay-Malabry, France

5 b Institut Galien Paris-Sud, CNRS, Univ. Paris-Sud, Université Paris-Saclay, Châtenay-Malabry,

6 France

7 * Corresponding author: Séverine Péchiné

$8 \quad$ E-mail address: $\underline{\text { severine.pechine@u-psud.fr }}$

9 Postal Address: Université Paris-Sud, UFR de Pharmacie, 5 rue J.B. Clément, 92296 Chatenay-

10 Malabry cedex

11 Abstract

12 Clostridium difficile flagellin FliC is a highly immunogenic pathogen-associated molecular pattern

13 playing a key role in $C$. difficile pathogenesis and gut colonization. Here, we designed an oral vaccine

14 against $C$. difficile with FliC encapsulated into pectin beads for colonic release. Bead stability and

15 FliC retention was confirmed in vitro using simulated intestinal media (SIM), while bead degradation

16 and FliC release was observed upon incubation in simulated colonic media (SCM). The importance

17 of FliC encapsulation into pectin beads for protection against $C$. difficile was assessed in a

18 vaccination assay using a lethal hamster model of $C$. difficile infection. Three groups of hamsters

19 orally received either FliC-loaded beads or unloaded beads in gastro-resistant capsule to limit gastric

20 degradation or free FliC. Two other groups were immunized with free FliC, one intra-rectally and the 
21 other intra-peritoneally. Hamsters were then challenged with a lethal dose of C. difficile VPI 10463.

22 Fifty percent of hamsters orally immunized with FliC-loaded beads survived whereas all hamsters

23 orally immunized with free FliC died within 7 days post challenge. No significant protection was

24 observed in the other groups. Only intra-peritoneally immunized hamsters presented anti-FliC $\mathrm{IgG}$

25 antibodies in sera after immunizations. These results suggest that an oral immunization with FliC-

26 loaded beads probably induced a mucosal immune response, therefore providing a protective effect.

27 This study confirms the importance of FliC encapsulation into pectin beads for a protective oral 28 vaccine against $C$. difficile.

29 Keywords: Clostridium difficile; oral vaccination; flagellin; pectin beads; colonic delivery

\section{$31 \quad 1 \quad$ Introduction}

32 Clostridium difficile (Clostridioides) difficile [1] is a Gram-positive, anaerobic spore-forming

33 bacterium and is the leading cause of antibiotic-associated diarrhea. Gut microbiota dysbiosis enables

34 C. difficile colonization of the intestinal tract. After contamination, C. difficile spores germinate,

35 vegetative forms multiply, and toxins are released, disrupting epithelium integrity and inducing an

36 inflammatory response in the colon [2]. C. difficile is a non-invasive pathogen, thus, promoting local

37 intestinal immunity could trigger early protection against $C$. difficile infection (CDI) [3]. Even

38 though the intra-rectal route has shown promising results previously by inducing a protective

39 immune response directed to $C$. difficile [4-6], developing the oral route of immunization

40 represents a rational choice to induce a gut mucosal immune response with a better patient

41 acceptance and comfort. To overcome the gastrointestinal barrier, antigen encapsulation is

42 recommended to maintain its integrity and its immunogenicity. Biocompatible and biodegradable

43 polymers are interesting materials for encapsulation. For instance, pectin, a non-toxic polysaccharide, 
44 has previously shown promising capacity for protein encapsulation and colonic delivery [7, 8].

45 Indeed, pectin is not degraded by gastric or intestinal enzymes but is almost totally degraded by

46 pectinolytic enzymes produced by the microbiota present in the colon $[7,9]$.

47 Current strategies for vaccine development against $C$. difficile target either toxins or colonization

48 factors. Systemic vaccines targeting toxins are currently being tested in clinical trials

49 (NCT01887912; NCT03090191; NCT02316470) and have shown efficacy in protecting against CDI

50 in animal models [10, 11]. However, targeting colonization factors could prevent $C$. difficile

51 colonization, growth and symptomatic infection therefore limiting dissemination of the bacteria in

52 the environment. Several vaccine candidates targeting colonization factors showed promising results

$53[4,8,12]$. For instance, flagellin which is highly immunogenic, harbors a unique pathogen-associated

54 molecular pattern implicated in toll-like receptor-5 (TLR-5) recognition. However, the central

55 domain of the flagellin is highly variable among different species [13, 14]. Regarding C. difficile

56 flagellin, FliC is a $39 \mathrm{kDa}$ structural protein of the flagellum. The central domain, the $\mathrm{N}$ - and C-

57 terminal domains of FliC are well conserved among the different strains of C. difficile. FliC

58 antibodies have been detected in patient sera [15] and non-CDI patients presented significantly more

59 anti-FliC serum antibodies than CDI patients [16]. Interestingly, the immunological properties of

60 FliC enable it to act as adjuvant [17] and as antigen, so, FliC represents a promising vaccine

61 candidate $[12,18]$. Ghose et al. intraperitoneally immunized hamsters and mice with FliC and

62 described the induction of a systemic IgG response protective against CDI [12].

63 Developing a mucosal vaccine targeting $C$. difficile gut colonization could enable early

64 protection against CDI. Oral vaccines can be expected to have much greater acceptability than

65 injectable vaccines. Oral vaccine administration could also lead to simplified manufacturing

66 vaccine, thereby increasing the potential for local vaccine production. Here we describe the

67 development and the efficacy of a mucosal vaccine strategy with FliC against $C$. difficile virulence in 
hamster. To this end, here we first assess the suitability of pectin beads to deliver FliC into the colon

69 and we evaluate FliC-loaded pectin beads in gastro-resistant capsules as an oral vaccine candidate

70 against $C$. difficile in a lethal hamster model of $\mathbf{C D I}$.

\section{Material and methods}

$72 \quad 2.1$ Preparation of pectin beads

73 Recombinant FliC was obtained and purified as previously described [13] with exception that the 74 purified protein was dialyzed against TRIS buffer $(25 \mathrm{mM}, \mathrm{pH} 7.5)$. Pectin beads were prepared as

75 previously detailed [8], pectin solution was obtained with Unipectine ${ }^{\mathrm{TM}}$ OG175C (Cargill) dissolved 76 in TRIS buffer $(25 \mathrm{mM}, \mathrm{pH} 7.5)$ at the concentration of $6 \%(\mathrm{w} / \mathrm{V})$. Unloaded beads were prepared 77 with the pectin solution only and FliC-loaded beads with pectin solution and recombinant FliC. The 78 pectin solution containing FliC or not was then dropped into a cross-linking solution of $\mathrm{Zn}$ acetate $79(12 \%, w / V)$. Beads, formed instantaneously by contact with zinc ions, were left in the cross-linking 80 solution for 30 minutes at room temperature under magnetic stirring. Beads were washed three times 81 with distilled water and then dried 3 hours at $37^{\circ} \mathrm{C}$. To determine encapsulation efficiency (EE), 82 FliC-loaded beads were disintegrated in TRIS buffer (25 mM, pH 7.5) added with EDTA (50 mM).

83 Then, proteins were dosed using Bradford dye-binding method. EE(\%) was determined by the

84 following formula : (actual amount of FliC encapsulated / theoretical amount of FliC 85 encapsulated) $* \mathbf{1 0 0}$.

$86 \quad 2.2$ Analysis of protein release from beads in simulated digestive media

87 Analysis of FliC release was carried out in two different simulated digestive media as previously described [8]: 5 h in Simulated Intestinal Medium (SIM, HEPES/NaCl buffer, pH 6.8) containing

89 pancreatin $(1 \% \mathrm{w} / \mathrm{V})$ followed by $5 \mathrm{~h}$ in Simulated Colonic Medium (SCM, HEPES/NaCl buffer, $\mathrm{pH}$

90 6) containing $\geq 760 \mathrm{U} / \mathrm{mL}$ of pectinase from Aspergillus aculeatus (Sigma). Presence of FliC and 
91 pectinase in the same medium makes difficult to dose proteins by Bradford dye-binding method.

92 Therefore, loaded beads were prepared using rhodamine-labelled FliC. Labeling and release protocol

93 were performed as previously described [8] and the amount of FliC released was determined by

94 spectrofluorometry in duplicate.

95 2.3 Gastro-resistant coating of capsules containing beads for oral immunization

96 To protect beads from the harsh gastric acid environment, loaded and unloaded beads were placed

97 into gelatin capsules (size 9, Harvard Apparatus) coated with a hydroxypropyl methylcellulose

98 phthalate (HPMCP 50) film. Coating was performed by dipping capsules into a $10 \%$ (w/V) solution

99 of HPMCP 50 in acetone/ethanol $(1: 1 \mathrm{v}: \mathrm{v})$ and drying them at room temperature [8]. This process

100 was repeated six times. The efficacy of the gastro-resistant coating was checked by a disintegration

101 test according to European pharmacopoeia (capsules undamaged after $2 \mathrm{~h}$ in $\mathrm{HCl} 0.1 \mathrm{M}$ ) [8].

$102 \quad 2.4$ Animals

103 The vaccination study was carried out in a model of infection using Mesocricetus auratus female

104 hamsters (weight, 80-100 g, Janvier Laboratories). Protocols involving animals and their care were

105 conducted in conformity with the institutional guidelines that are in compliance with national and

106 international laws and policies. The protocol was approved by the Committee on the Ethics of

107 Animal Experiments University of Paris-Sud and the French Minister of Research (APAFIS\#4577-

$1082016020913152994 \mathrm{v} 4)$. All efforts were made to minimize animal suffering.

$109 \quad 2.5 \quad$ Vaccination protocol

110 Five groups of 6 animals were used. Each vaccinated animals received a total of $300 \mu \mathrm{g}$ of FliC in

111 three administrations of $100 \mu \mathrm{g}$ of FliC every fifteen days (at day 0, day 15 and day 30). One group

112 was immunized with FliC-loaded pectin beads given orally and a group orally received unloaded 
113 beads. Three other groups were immunized with recombinant free FliC by oral, intra-rectal or intra-

114 peritoneal route. Fifteen days after the last immunization (at day 45) and before C. difficile

115 challenge, hamsters were orally given clindamycin at a single dose of $50 \mathrm{mg} / \mathrm{kg}$ and gentamicin twice

116 a day during 5 days (from day 45 to day 49) at a dose of $2.5 \mathrm{mg} / \mathrm{kg}$ to disrupt the intestinal

117 microbiota. Then at day 50, hamsters were orally challenged with $7,5 \times 10^{4}$ spores of $C$. difficile strain

118 VPI 10463 (Fig. 1). Two days after challenge, $\boldsymbol{C}$. difficile colonization was checked by detection of

119 C. difficile vegetative cells in the feces by plating adequate dilutions on Columbia agar

120 containing $5 \%$ of horse blood, $25 \%(w / v)$ of $D$-cycloserine, and $0.8 \%(w / v)$ of cefoxitin and

121 taurocholate.

122 Evaluation of specific antibody level in sera after immunization

123 To evaluate the antibody response in sera, blood samples were withdrawn before the first

124 immunization and 15 days after the last immunization, before $C$. difficile challenge. Indirect ELISA

125 was used to detect antibodies in the sera as previously described [6]. Briefly, wells of 96-well

126 microtitre plates (MaxiSorp, Nunc) were coated with $100 \mu \mathrm{L}$ of a $5 \mu \mathrm{g} / \mathrm{mL}$ solution of recombinant

127 purified FliC. Sera were tested in duplicate at dilution 1:500 in 100 $\mu \mathrm{L}$ final volume. After five

128 washings with phosphate buffer (PBS) and Tween-20 (0.1\%), an aliquot of $100 \mu \mathrm{L}$ per well of a

129 rabbit anti-hamster IgG conjugated with biotin (1:8,000 dilution; Biovalley) was added and plates

130 were incubated for $30 \mathrm{~min}$ at $37^{\circ} \mathrm{C}$. Then, after five washings, $100 \mu \mathrm{L}$ per well of streptavidin-HRP

131 (1:10,000 dilution; ThermoScientific) were added and plates were incubated for 30 min at $37^{\circ} \mathrm{C}$.

132 Assays with antigen in the absence of sera served as negative controls. Immunoglobulin levels are 133 expressed as OD units at $450 \mathrm{~nm}$.

$134 \quad 2.7 \quad$ Statistical analysis 
135 Animal surviving rate was analyzed using Kaplan-Meier estimates. Survival rates between groups

136 were compared using log rank test, p-values $<0.05$ were considered as statistically significant.

137 Mann-Withney U-test was performed to analyzed specific anti-FliC antibody levels in sera after

138 immunizations, p-values $<0.05$ were considered as statistically significant.

\section{$1393 \quad$ Results}

$140 \quad 3.1 \quad$ Flagellin encapsulation in pectin beads and in vitro release

141 In order to perform oral vaccination, we encapsulated the recombinant $C$. difficile flagellin FliC into

142 pectin beads. About 40 to 45 beads were obtained per milliliter of pectin solution. After drying, beads

143 had an ellipsoid shape with a 1.2-1.5 mm diameter and weighed between 1.5 and $2 \mathrm{mg}$ (Fig. 2). The

144 total amount of encapsulated FliC was dosed after total disintegration of beads. FliC-loaded bead

145 contained a total amount of $3 \pm 1 \mu \mathrm{g}$ of FliC by bead, this corresponds to an encapsulation efficiency

146 of about $30 \pm 10 \%(n=5)$. In vitro characterization confirms beads stability in SIM and protein release

147 in SCM containing pectinase. Less than $10 \%$ of FliC was released after $5 \mathrm{~h}$ of incubation in SIM and

148 dried beads swelled keeping their shape. After being placed in SCM, beads started to disintegrate

149 leading to the release of FliC. Between 2 and $3 \mathrm{~h}$ were sufficient to release more than $80 \%$ of

150 entrapped FliC (Fig. 3). This in vitro characterization of FliC-loaded beads confirms the interest of

151 using this encapsulation strategy for colonic delivery of an antigen after oral administration.

$152 \quad 3.2$ Vaccination with flagellin against $\mathbf{C D I}$ in the hamster model

153 C. difficile flagellin FliC is a promising vaccine candidate. To evaluate the efficacy of mucosal

154 vaccination with FliC against $C$. difficile virulence, we immunized hamsters with the same amount of

155 FliC via different mucosal routes and compare them with the intra-peritoneal parenteral route. We

156 showed here that encapsulation of FliC into pectin beads for oral administration significantly 
157 protected hamsters against $C$. difficile lethal challenge (Fig. 4). Indeed, at day 17 after challenge,

$15850 \%$ of hamsters survived in the group orally vaccinated with FliC-loaded beads, whereas no survival

159 was observed after oral vaccination with free FliC (p-value=0.041). Only 17\% of hamsters $(n=1)$

160 survived in the unloaded bead group. The same percentage of surviving animals was observed for the

161 intra-rectally vaccinated group. After immunization by intra-peritoneal route, $33 \%$ of hamsters

162 survived. Interestingly, analysis of $C$. difficile fecal shedding showed that, whereas all 30 animals

163 were infected by $C$. difficile two days post-challenge, all surviving animals in the different groups

164 were no more colonized at the end of the assay at 17 days post challenge. These results showed that

165 oral vaccination with FliC-loaded pectin beads led to the best protection against $C$. difficile virulence

166 in the hamster model.

$167 \quad 3.3 \quad$ Antibody response induced after vaccination

168 To correlate the observed protection with the systemic immune response induced by vaccination, we

169 evaluated the anti-FliC response in sera. We observed an increased level of FliC-specific IgG

170 antibodies in sera after intra-peritoneal immunizations of hamsters. In contrast, in the other groups,

171 no significant increase of anti-FliC-specific serum IgG level was observed (Fig. 5).

\section{Discussion}

173 As $C$. difficile is a non-invasive enteropathogen, the induction of a mucosal immune response close

174 to the site of infection appears to be a relevant choice for inducing protection. Vaccine development 175 against non-invasive gastrointestinal infections such as enterotoxigenic Vibrio cholerae or

176 Escherichia coli showed that the protection is conferred mainly by specific secretory $\operatorname{IgA}(\operatorname{sg} \mathrm{A})$ and

177 by the induction of a memory immune response [19]. A mucosal vaccine strategy, which aims to 178 prevent alteration of the intestinal epithelium by targeting the early stages of the $C$. difficile infectious 179 process, could benefit from further attention. 
180 Furthermore, one of the vaccine strategies against $C$. difficile is to target surface proteins involved in

181 intestinal colonization. The flagellin FliC appears a promising vaccine candidate, since Ghose et al.

182 reported that intra-peritoneal immunization with FliC and alum led to 43 to $64 \%$ of protection in a

183 hamster model and 40 to $100 \%$ protection in a mouse model in a dose-dependent manner [12]. In our

184 assay, intra-peritoneal immunization of hamsters with FliC led to 33\% of survival against C. difficile.

185 Compared to Ghose et al results [12], this difference of protection is probably due to the adjuvant

186 used for immunization. Indeed, as already demonstrated, $C$. difficile flagellin FliC has adjuvant

187 properties, avoiding alum use as adjuvant $[12,17]$. Consequently in our study, immunizations were

188 performed without additional adjuvant. In addition, $C$. difficile strain used for challenge was not the

189 same, with probably a difference of virulence between the VPI 10463 strain used here and the

$190630 \Delta e r m$ strain used in Ghose et al study, as described elsewhere [20]. Of note here, we

191 demonstrated that the high immunogenicity of FliC enables to induce a strong systemic IgG antibody

192 response after intra-peritoneal immunization without supplementary adjuvant.

193 Interestingly, here we obtained a better protection with an oral vaccine consisting of FliC

194 encapsulated into pectin beads to target colonic release than after intra-peritoneal immunization with

195 purified FliC alone. We showed that this strategy can significantly protect $50 \%$ of hamsters from

196 CDI (3 out of 6). However, these results should be confirmed in a larger study. This significant

197 but partial protection confirms the multifactorial aspect of colonization, suggesting that a

198 combination of several proteins will be necessary to trigger an efficient immune response

199 against $\boldsymbol{C}$. difficile colonization factors, and consequently to prevent the colonization process. In

200 addition, according to animal variability to $C$. difficile infection, especially regarding immune

201 response and microbiota, a combined vaccine could be necessary.

202 Ghose et al. demonstrated that the protection induced by FliC immunization by intra-peritoneal route

203 was anti-FliC IgG-mediated. In our study, the immune response induced after oral immunization with 
204 FliC-loaded pectin beads is able to partially protect animal from death. However, no specific IgG 205 antibody response was detected in sera. This was previously observed in another assay of oral 206 vaccination of hamsters with the Cwp84 protease encapsulated in pectin beads. Although vaccinated 207 hamsters were partially protected (40\%) against CDI, they did not develop a systemic anti-Cwp84208 IgG antibody response [8]. This suggests that after vaccination by mucosal route, beside a systemic 209 immune response, a local immune response with sIgA production could be the key factor of 210 protection. It has been previously shown in a mouse model that parenteral immunization with 211 flagellin can activate mucosal dendritic cells and induce an isotype switch to IgA [18]. Unfortunately 212 secondary antibodies are not commercially available to detect specific IgA in hamsters.

213 Here, we used the hamster model of CDI, which is highly sensitive to this infection and reflects more 214 severe infection in human than mild infection. Our results indicate that in this model, protection 215 could probably be related to neutralizing SIgA but other factors may play an important role in the 216 host immune response against CDI. In particular, our immunization strategy might have generated a 217 wider cell-based immunity that could have induced partial protection. Regarding Streptococcus 218 pneumoniae, it has been demonstrated that multiple immune cell types are required for the induction

219 of a protective immunity in a murine model which lacks mature B cells and fails to produce antibody 220 [21]. Further studies are needed to specify the immune effectors induced by immunization.

221 In this study, the least protection of hamsters observed by intra-rectal administration of FliC 222 compared to the oral administration of FliC-loaded beads and the absence of protection for the free 223 FliC orally treated group, is presumably due to the degradation of the free antigen by gut enzymes 224 before it reaches the colon. This further confirms the importance of the administration route and the 225 use of pectin beads as a delivery system for FliC. 
226 To conclude, we showed that oral vaccination with $C$. difficile FliC-loaded pectin beads is partially

227 protective against a virulent strain of $C$. difficile in a hamster model. In order to assess in further

228 depth the mechanisms of protection, further studies in mouse model could inform on the

229 protection against $\boldsymbol{C}$. difficile colonization and a better understanding of the immune response

230 elicited with this vaccine. This study confirms the importance of the adequacy between the

231 administration route, the delivery system and the vaccine candidate in the design of a mucosal

232 protective immunization strategy targeting $C$. difficile.

\section{Acknowledgments}

234 This work was supported by the technical assistance of Valerie Dupont-Domergue and staff from the 235 animal care facility of IPSIT.

\section{Funding}

237 Jean-François Bruxelle's PhD was funded by the French Ministry of Research and Higher Education

\section{Conflict of interest}

239 No conflicts of interest to declare. 

Blood sample

- D45 Clindamycin 50mg/kg

3 immunizations every 15 days - Gentamicin 2,5 mg/kg

$2 X$ per day during 5 days
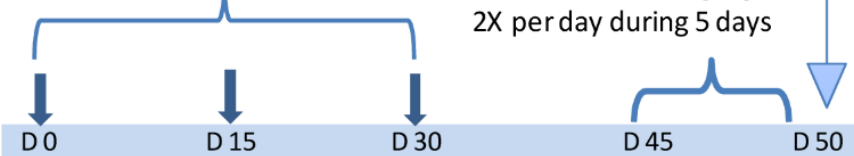

Survival monitoring

D 45

D 50

243 Figure 1. Vaccination protocol.

244 Animals received a total of $300 \mu \mathrm{g}$ of FliC in three administrations of $100 \mu \mathrm{g}$ of FliC on days 0,15

245 and 30. Before challenge, hamsters received clindamycin and gentamicin to disrupt the intestinal

246 microbiota. Then, hamsters were orally challenged by $7,5 \times 10^{4}$ spores of $C$. difficile strain VPI 10463.

247 Two days after challenge CDI was checked by detection of $C$. difficile in the feces. Blood samples

248 were withdrawn before the first immunization and 15 days after the last immunization to evaluate the

249 antibody response in sera.

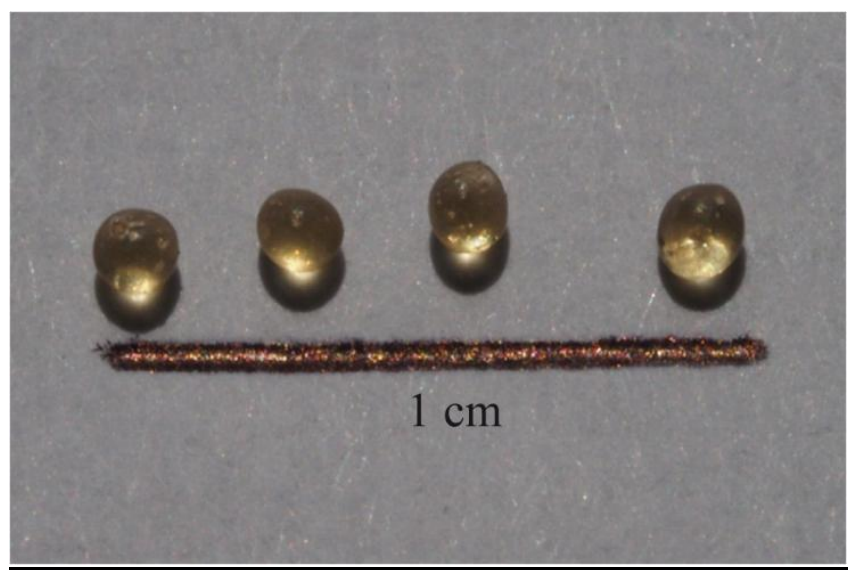

253 Figure 2. Dried pectin beads (scale bar represents $1 \mathrm{~cm}$ ). 


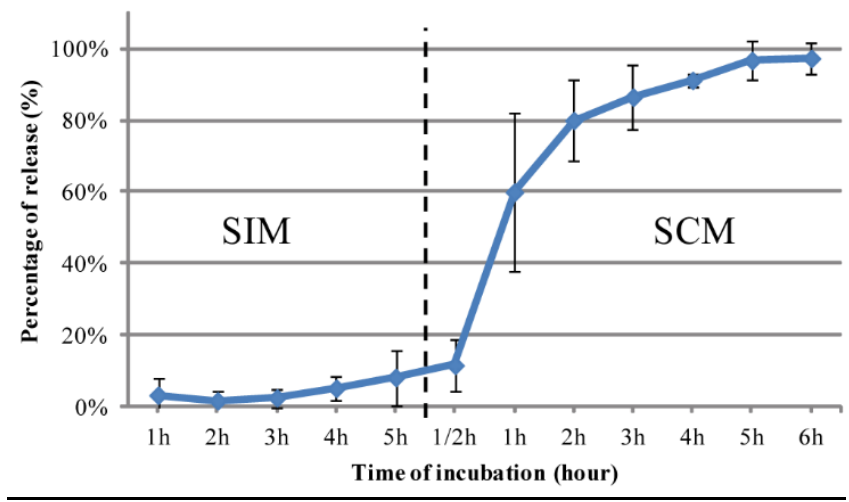

255 Figure 3. In vitro FliC release from pectin beads in Simulated Intestinal Medium (SIM) for 5h and 256 Simulated Colonic Medium (SCM) for other 5h $(\mathrm{n}=2)$.

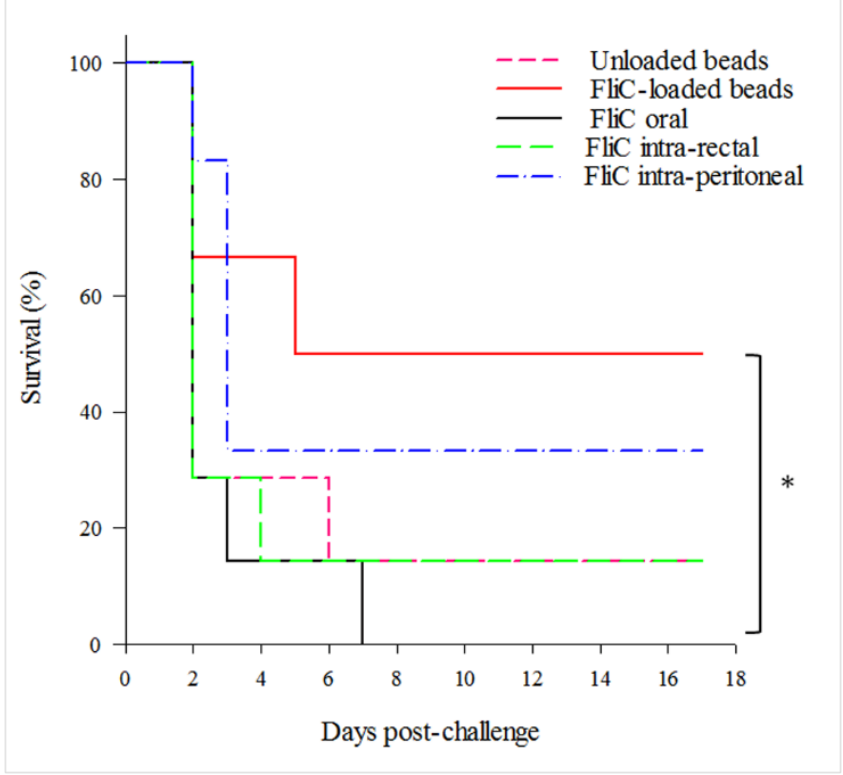

258 Figure 4. Kaplan-Meier survival estimates after immunizations. One group was immunized with

259 FliC-loaded beads given orally (FliC-loaded beads), one group orally received unloaded pectin beads

260 (Unloaded beads) and three other groups were immunized with recombinant free FliC by oral (FliC

261 oral), intra-rectal (FliC intra-rectal) or intra-peritoneal route (FliC intra-peritoneal). After challenge

262 with spores of $C$. difficile VPI 10463 strain, animals were monitored for 17 days. * p-value $<0.05$ 


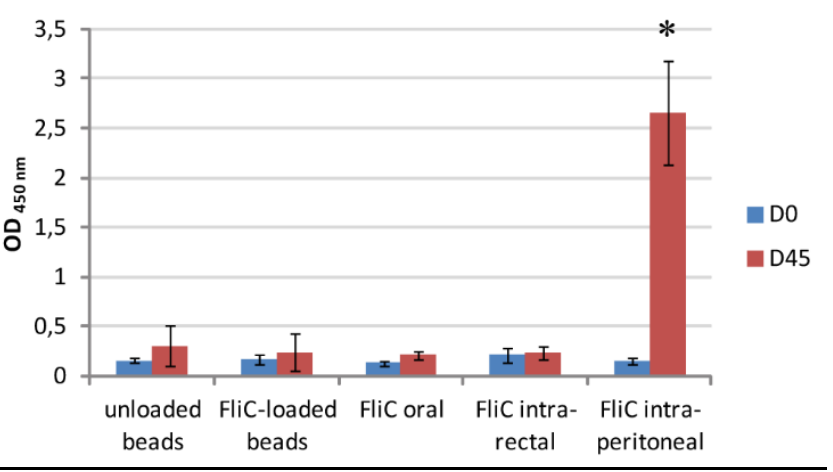

264 Figure 5. Detection of specific IgG in animal sera by ELISA. Mean of anti-FliC-specific IgG in

265 serum of immunized hamsters (diluted 1:500) before (D0) and after vaccination (D45). Sera of 266 hamsters were analysed by ELISA. *: statistically significant difference p-value $<0.05$ (Mann267 Withney U-test).

\section{References}

276 [1] Lawson PA, Citron DM, Tyrrell KL, Finegold SM. Reclassification of Clostridium difficile as 277 Clostridioides difficile (Hall and O'Toole 1935) Prévot 1938. Anaerobe 2016 Aug 2016;40:95-9.

278 [2] Koenigsknecht MJ, Theriot CM, Bergin IL, Schumacher CA, Schloss PD, Young VB.

279 Dynamics and Establishment of Clostridium difficile Infection in the Murine Gastrointestinal Tract.

280 Infection and Immunity 2015 03/2015;83:934-41. 
281 [3] Péchiné S, Collignon A. Immune responses induced by Clostridium difficile. Anaerobe 2016 282 Oct 2016;41:68-78.

283 [4] Bruxelle J-F, Mizrahi A, Hoys S, Collignon A, Janoir C, Péchiné S. Immunogenic properties 284 of the surface layer precursor of Clostridium difficile and vaccination assays in animal models.

285 Anaerobe 2016 Feb 2016;37:78-84.

286 [5] Mizrahi A, Collignon A, Péchiné S. Passive and active immunization strategies against

287 Clostridium difficile infections: state of the art. Anaerobe 2014 Dec 2014;30:210-9.

288 [6] Péchiné S, Denève C, Le Monnier A, Hoys S, Janoir C, Collignon A. Immunization of 289 hamsters against Clostridium difficile infection using the Cwp84 protease as an antigen. FEMS 290 Immunology \& Medical Microbiology 2011 10/2011;63:73-81.

291 [7] Bourgeois S, Laham A, Besnard M, Andremont A, Fattal E. In vitro and in vivo evaluation of 292 pectin beads for the colon delivery of beta-lactamases. Journal of Drug Targeting 2005 Jun 293 2005; 13:277-84.

294 [8] Sandolo C, Péchiné S, Le Monnier A, Hoys S, Janoir C, Coviello T, et al. Encapsulation of 295 Cwp84 into pectin beads for oral vaccination against Clostridium difficile. 2011 Nov 2011;79:56629673.

297 [9] Ndeh D, Rogowski A, Cartmell A, Luis AS, Basle A, Gray J, et al. Complex pectin 298 metabolism by gut bacteria reveals novel catalytic functions. Nature 2017 Apr 6;544(7648):65-70.

299 [10] Anosova NG, Brown AM, Li L, Liu N, Cole LE, Zhang J, et al. Systemic antibody responses 300 induced by a two-component Clostridium difficile toxoid vaccine protect against C. difficile301 associated disease in hamsters. Journal of Medical Microbiology 2013 Sep 2013;62:1394-404.

302 [11] Kociolek LK, Gerding DN. Breakthroughs in the treatment and prevention of Clostridium 303 difficile infection. Nature Reviews Gastroenterology \& Hepatology 2016 Mar 2016;13:150-60.

304 [12] Ghose C, Eugenis I, Sun X, Edwards AN, McBride SM, Pride DT, et al. Immunogenicity and 305 protective efficacy of recombinant Clostridium difficile flagellar protein FliC. Emerging Microbes \& 306 Infections 2016 Feb 03, 2016;5:e8.

307 [13] Batah J, Denève-Larrazet C, Jolivot P-A, Kuehne S, Collignon A, Marvaud J-C, et al.

308 Clostridium difficile flagella predominantly activate TLR5-linked NF- $\kappa$ B pathway in epithelial cells. 309 Anaerobe 2016 Apr 2016;38:116-24.

310 [14] Rumbo M, Nempont C, Kraehenbuhl J-P, Sirard J-C. Mucosal interplay among commensal 311 and pathogenic bacteria: Lessons from flagellin and Toll-like receptor 5. FEBS Letters $20062006-$ 312 05-22;580:2976-84.

313 [15] Wright A, Drudy D, Kyne L, Brown K, Fairweather NF. Immunoreactive cell wall proteins of 314 Clostridium difficile identified by human sera. Journal of medical microbiology 2008 Jun 315 2008;57:750-6.

316 [16] Péchiné S, Gleizes A, Janoir C, Gorges-Kergot R, Barc M-C, Delmée M, et al. 317 Immunological properties of surface proteins of Clostridium difficile. Journal of medical 318 microbiology 2005 Feb 2005;54:193-6.

319 [17] Bruxelle J-F, Mizrahi A, Hoÿs S, Collignon A, Janoir C, Péchiné S. Clostridium difficile 320 flagellin FliC: Evaluation as adjuvant and use in a mucosal vaccine against Clostridium difficile. PloS 321 One 2017 2017;12:e0187212. 
322 [18] Flores-Langarica A, Marshall JL, Hitchcock J, Cook C, Jobanputra J, Bobat S, et al. Systemic

323 flagellin immunization stimulates mucosal CD103+ dendritic cells and drives Foxp3+ regulatory T

324 cell and IgA responses in the mesenteric lymph node. Journal of Immunology (Baltimore, Md: 1950)

3252012 Dec 15, 2012;189:5745-54.

326 [19] Holmgren J, Czerkinsky C. Mucosal immunity and vaccines. Nature Medicine 2005 Apr

327 2005; 11:S45-53.

328 [20] Theriot CM, Koumpouras CC, Carlson PE, Bergin II, Aronoff DM, Young VB.

329 Cefoperazone-treated mice as an experimental platform to assess differential virulence of Clostridium

330 difficile strains. Gut Microbes 20112011 Nov-Dec;2:326-34.

331 [21] McCool TL, Weiser JN. Limited role of antibody in clearance of Streptococcus pneumoniae

332 in a murine model of colonization. Infect Immun 2004 Oct;72(10):5807-13. 